\title{
Complex renal masses: partial or no partial nephrectomy?
}

\author{
Riccardo Lombardo ${ }^{1}$, Costantino Leonardo ${ }^{2}$, Anton Zarraonandia ${ }^{3}$, Andrea Tubaro ${ }^{1}$, Cosimo De Nunzio ${ }^{1}$ \\ ${ }^{1}$ Department of Urology, Ospedale Sant'Andrea, 'Sapienza' University of Rome, Rome, Italy; ${ }^{2}$ Department of Urology, Policlinico Umberto I, \\ 'Sapienza' University of Rome, Rome, Italy; ${ }^{3}$ Department of Urology, Hospital de Montecelo, Pontevedra, Spain \\ Correspondence to: Riccardo Lombardo, MD. Department of Urology, University "La Sapienza", Via di Grottarossa 1039, 00189 Rome, Italy. \\ Email: rlombardo@me.com. \\ Provenance: This is an invited article commissioned by the Section Editor Dr. Xiao Li (Department of Urology, Jiangsu Cancer Hospital, Jiangsu \\ Institute of Cancer Research, Nanjing Medical University Affiliated Cancer Hospital, Nanjing, China). \\ Comment on: Beksac AT, Okhawere KE, Elbakry AA, et al. Management of high complexity renal masses in partial nephrectomy: A multicenter \\ analysis. Urol Oncol 2019;37:437-44.
}

Submitted Sep 23, 2019. Accepted for publication Sep 30, 2019.

doi: $10.21037 / \mathrm{atm} .2019 .10 .03$

View this article at: http://dx.doi.org/10.21037/atm.2019.10.03

Management of high complexity renal masses is always challenging for urologists. Beksac and co-workers made a great effort in collecting data from 144 patients with complex renal masses defined as a RENAL score higher than 10 undergoing robotic partial nephrectomy (RPN). Trifecta was achieved in $62 \%$ of the patients. The multi-center design of the study is an important feature considering that 6 surgeons were involved. The study is retrospective and follow up is short therefore data should be managed with caution. Their study confirms the available evidence on the subject, confirming that in expert hands RPN should be performed whenever deemed feasible.

In front of a renal mass the main objectives of surgeons are to achieve a good oncological outcomes, preserve renal function and avoid complications.

The oncological outcomes of Beksac series are in line with the available literature with a rate of positive surgical margins (PSM) and recurrence of 5\% and 7\% respectively. Some studies suggest PSM may translate into negative oncological outcomes, however most of these studies are retrospective and include open, laparoscopic and robotic interventions. According to the latest EAU guidelines the role of PSM is still unknown as they do not translate into a higher tendency towards the development of metastases or a decreased cancer-specific survival (CSS). These patients should undergo a closer follow up as reintervention may result in overtreatment (1).

In terms of functional outcomes, in Beksac cohort median warm ischemia time (WIT) was $20 \mathrm{~min}$ and $20 \%$ of the patients developed de novo chronic kidney disease after surgery. According to the available literature renal function preservation relies on the amount of renal parenchyma preserved and on WIT which should be under $20 \mathrm{~min}$ (2). Notwithstanding the complexity of the cases, surgeons in Beksac cohort have the merit of achieving very good functional outcomes after RPN. Although the excellent results of the study are biased by a short follow up (6 months).

Complication rate of Beksac series is $17 \%$ with a major complication rate of $2 \%$ which is perfectly in line with the available series on PRN. As well only 1 patient needed transfusion and no conversion to open surgery was needed.

R.E.N.A.L and PADUA scores have been developed in order to assess in an objective manner the risk of renal surgery (3). However both scores do not take into consideration the expertise of the surgeon who is performing the surgery. Very recently Buffi et al. performed a similar study assessing the outcomes of patients undergoing RPN with PADUA scores above 10 (4). The rate of optimal surgical outcomes was $62 \%$ which is identical to the present study. Oncological, functional and complication outcomes were as well comparable to Beksac study. In the era of evidence-based medicine the role of the surgeon is a difficult variable to assess. Very often the ability of the surgeon is measured on the number of cases performed which probably is not a truly reliable measure. Recently surgeons have been compared to high level professional sport players which are definitely not judged on number of games played. An expert surgeon should be defined on its experience, knowledge, 
technical and more importantly non-technical skills $(5,6)$. Probably in the near future technology and artificial intelligence will better define an expert surgeon which can objectively approach complex renal masses.

Beksac study adds evidence on the feasibility of approaching complex renal masses with robot assisted technology. Up to date, the available data is mainly retrospective which severely limits the conclusions on the subject. In the future, prospective data is essential to clearly define outcomes of complex renal masses RPN. Up to date, surgery of complex renal masses should be exclusively performed in experienced centers by experienced surgeons. An important question is what could improve these outcomes in the near future?

An important area of research which may help surgeons when planning renal surgery is $3 \mathrm{D}$ printing. Porpiglia et al. evaluated the use of these models and concluded that $3 \mathrm{D}$ printing technology has been perceived as a useful tool for the purpose of surgical planning, physician education/ training and patient counselling (7). As well as nearinfrared fluorescence imaging with indocyanine green is a potential technology which may improve functional outcomes in partial nephrectomy. A recent meta-analysis by Veccia et al. (8) suggests patients undergoing NIRF PN have better short-term functional outcomes when compared to patients undergoing classic $\mathrm{PN}$ [weighted mean difference (WMD): $9.26 \mathrm{~mL} / \mathrm{min} ; 95 \%$ confidence interval (CI): 6.46, 12.06; $\mathrm{P}<0.001]$. Both $3 \mathrm{D}$ printing and NIRF as still to be considered investigational and future studies should better define the role of these new technologies in PN.

In conclusion, RPN should be performed in expert centers whenever deemed possible. Future studies should investigate methods to assess surgical expertise and skills needed to perform RPN in complex renal masses.

\section{Acknowledgments}

None.

\section{Footnote}

Conflicts of Interest: The authors have no conflicts of interest to declare.

Ethical Statement: The authors are accountable for all aspects of the work in ensuring that questions related to the accuracy or integrity of any part of the work are appropriately investigated and resolved.

\section{References}

1. Marszalek M, Carini M, Chlosta P, et al. Positive surgical margins after nephron-sparing surgery. Eur Urol 2012;61:757-63.

2. Volpe A, Blute ML, Ficarra V, et al. Renal Ischemia and Function After Partial Nephrectomy: A Collaborative Review of the Literature. Eur Urol 2015;68:61-74.

3. Borgmann H, Reiss AK, Kurosch M, et al. R.E.N.A.L. Score Outperforms PADUA Score, C-Index and DAP Score for Outcome Prediction of Nephron Sparing Surgery in a Selected Cohort. J Urol 2016;196:664-71.

4. Buffi NM, Saita A, Lughezzani G, et al. Robot-assisted Partial Nephrectomy for Complex (PADUA Score $\geq 10$ ) Tumors: Techniques and Results from a Multicenter Experience at Four High-volume Centers. Eur Urol 2020;77:95-100.

5. Lubowitz JH, Provencher MT, Brand JC, et al. Expert Surgeons Can Be Distinguished From Trainees, and Surgical Proficiency Can Be Defined, Using Validated Metrics and Shoulder Models. Arthroscopy 2015;31:1635-6.

6. Gjeraa K, Spanager L, Konge L, Petersen RH, et al. Nontechnical skills in minimally invasive surgery teams: a systematic review. Surg Endosc 2016;30:5185-99.

7. Porpiglia F, Bertolo R, Checcucci E, et al. Development and validation of $3 \mathrm{D}$ printed virtual models for robotassisted radical prostatectomy and partial nephrectomy: urologists' and patients' perception. World J Urol 2018;36:201-7.

8. Veccia A, Antonelli A, Hampton LJ, et al. Near-infrared Fluorescence Imaging with Indocyanine Green in Robot-assisted Partial Nephrectomy: Pooled Analysis of Comparative Studies. Eur Urol Focus 2019. doi: 10.1016/ j.euf.2019.03.005.
Cite this article as: Lombardo R, Leonardo C, Zarraonandia A, Tubaro A, De Nunzio C. Complex renal masses: partial or no partial nephrectomy? Ann Transl Med 2019;7(Suppl 8):S312. doi: $10.21037 /$ atm.2019.10.03 